kungskräfte zwischen Brom und Fremdgas, nicht dagegen die zwischen Brom und Brom bemerkbar. Ahnlich liegt der Fall beim Jod, wo nach Messungen von G. Kortüm und G. Friedheim ${ }^{44}$ im Sichtbaren das Beer sche Gesetz gültig ist, dagegen bei etwa $2700 \AA$ eine ausgeprägte konzentrationsabhängige Assoziationsbande auftritt.

Merkwürdig ist dabei weiter, daß diese Wechselwirkungskräfte zwischen $\mathrm{Br}_{2}$ und Fremdmolekeln vom Druck weitgehend unabhängig sein müssen, wie sich aus der Tatsache ergibt, daß für Mischungen konstanter Zusammensetzung das B e e r sche Gesetz ebenfalls gültig ist. An eine normale Assoziation ist also nicht zu denken. Maßgebend für den Effekt ist demnach allein das Mischungsverhältnis Brom - Fremdgas. Möglicherweise könnte der Grund hierfür in einer gewissen Entmischung zu suchen sein, indem die Fremdgasmolekeln sich in bestimmter, von den Wechselwirkungskräften abhängiger Weise um jede Brommolekel anordnen. Das würde bedeuten, daß in derartigen Gemischen keine völlig statistische Verteilung der Molekeln vorliegt, wie

${ }_{44}$ Z. Naturforschg., erscheint demnächst. das auch von Flüssigkeitsgemischen vor allem durch die Arbeiten von K. L. Wolf und Schülern bekannt ist.

Über die Natur der Wechselwirkungskräfte lassen sich nach den vorliegenden Ergebnissen kaum Aussagen machen. Um Dipolkräfte scheint es sich nicht zu handeln, da die Wirkung der verschiedenen Gase keinen Zusammenhang mit dem Dipolmoment erkennen läßt. Eher könnte man daran denken, die L o n d o n schen Dispersionskräfte zur Erklärung heranzuziehen. Die geringe Wirkung von Argon ließe sich dadurch deuten, daß seine abgeschlossene Schale nur ein schwaches Kraftfeld besitzt; den starken Effekt von Wasserstoff könnte man vielleicht als eine Art Resonanzkopplung auffassen, die eine Vorstufe zur chemischen Reaktion bildet. Ungeklärt bleibt auch, daß der Fremdgaseffekt nur im sichtbaren Bandengebiet und nicht im Kontinuum auftritt. Untersuchungen in dieser Richtung sind geplant.

Der Steinheil-Spektrograph wurde uns von Hrn. Prof. B uten andt für unsere Messungen in freundlicher Weise zur Verfügung gestellt, wofür wir unseren herzlichsten Dank aussprechen.

\title{
Nachweis der Faserstruktur von Fullererde (Attapulgit) im Elektronenmikroskop
}

\author{
Von Joachim ENDELL \\ (Z. Naturforschg. 1, 646-649 [1946]; aus Braunschweig ${ }^{1}$ eingegangen am 16. August 1946)
}

\begin{abstract}
Die Untersuchung im Elektronenübermikroskop ergab, daß der Attapulgit aus Fasern von 250 bis $50 \AA \AA$ Durchmesser besteht.

Die geringe Dicke dieser Fasern bestätigt den Strukturvorschlag von B rad le y, der einen Aufbau der Kristalle aus amphibolartigen Ketten vorsieht, entgegen den meisten Tonmineralien, die Schichtgitter besitzen.

Die Oberfläche der Fasern errechnet sich zu ca. $150 \mathrm{~m}^{2} / \mathrm{g}$ und läßt die gute Bleichwirkung der natürlichen amerikanischen Fullererde verstehen.
\end{abstract}

I m Jahre 1935 gab J. de Lapparent² dem Mineral der amerikanischen Fullererde, das zuerst bei Attapulgus in Georgia (USA.) und bei Mormoiron (Frankreich) gefunden wurde, den Namen Attapulgit. Schon vorher hatten U. $\mathrm{H}$ of mann, K. Endell und D. Wil m ${ }^{3}$ darauf aufmerksam gemacht, daß es amerikanische

1 Die experimentellen Untersuchungen wurden am Anorganisch-Chemischen Institut der Technischen Hochschule Wien ausgeführt.
Fullererden gibt, deren Röntgenbild ein besonderes Mineral zeigt, das z. B. vom Montmorillonit, dem Mineral vieler Bleicherden, verschieden ist. Dieses Mineral erwies sich als identisch mit Attapulgit. Sehr ähnliche Mineralien wurden zur selben Zeit von H.Longchambon ${ }^{4}$ untersucht

2 C. R. hebd. Séances Acad. Sci. 201, 481 [1935], 202, 1728 [1936], 203, 596 [1937], 212, 971 [1941].

3 Angew. Chem. 47, 539 [1934].

4 C. R. hebd. Séances Acad. Sci. 201, 483 [1935]. 
und als dem Palygorskit von M.Fersmann verwandt beschrieben.

1937 vertrat P. F. Ke rr ${ }^{5}$ die Ansicht, daß sich die Angaben über die Auffindung eines speziellen Tonminerals Attapulgit nicht bewahrheitet, sondern daß röntgenographische Untersuchung, Bestimmung des Brechungsindex und chemische Analyse gezeigt hätten, daß das mit Attapulgit bezeichnete Mineral identisch mit quarzhaltigem Montmorillonit sei. In einer ausführlich angelegten Schrift verteidigte $L$ apparent ${ }^{6}$ auf Grund verschiedenster Untersuchungsmethoden seine Annahme, daß Attapulgit ein spezielles, vom Montmorillonit verschiedenes Mineral sei. Dies ist auch nach dem Röntgenbild und insbesondere dem Fehlen der innerkristallinen Quellung nicht zu bezweifeln. - Während Long ch a mbon ${ }^{7}$ den Namen Attapulgit für unnötig hielt, da das Mineral identisch mit Palygorskit sei, verteidigte Lapparent den Namen Attapulgit als Sammelbezeichnung für eine Mineralgruppe, unter die auch der Palygorskit als faseriges alkalifreies Mineral fällt. Bei der großen technischen Bedeutung der amerikanischen Fullererden, deren Mineral mit dem bei Attapulgus gefundenen identisch ist, erscheint der Name Attapulgit in Übereinstimmung mit Lapparent als zweckmäßigste Bezeichnung.

Lapparent vertrat die Ansicht, daß die Kristallstruktur des Attapulgits - trotz der faserigen Gestalt der Palygorskite - aus glimmerähnlichen Schichten aufgebaut sei. Demgegenüber kam W.F. B r a d l e y ${ }^{8}$ auf Grund einer Strukturanalyse zu dem Resultat, daß der Attapulgit eine monokline Faserstruktur mit amphybolartigen Ketten habe. Auf diese Möglichkeit hatte schon früher G. Nagel s ch mid t ${ }^{9}$ hingewiesen. Das Vorliegen einer Faserstruktur wäre um so interessanter, als die Tonmineralien bisher fast alle Schichtstruktur und blättchenartig ausgebildete Kristalle besitzen.

Keine der bis dahin angewandten Unter-

5 Amer. Mineralogist 22, 534 [1937].

- Z. Kristallogr., Kristallgeometr., Kristallphysik, Kristallchem. [Abt. A d. Z. Kristallogr., Mineral., Pe. trogr.] 97, 237; 98, 233 [1937]; Bull. Soc. franç. Minéral. 61, 253 [1938].

7 Bull. Soc. franç. Minéral. 60, 237 [1937]; 'Ass. scientifique et technique de Ceramique (Kongreß Juni 1938).

${ }^{8}$ Amer. Mineralogist 26, 405 [1941].

๑ Nature [London] 142, 114 [1938]. suchungsmethoden zeigt die äußere Struktur der feinsten Bestandteile so klar wie das Elektronenmikroskop. Es wurden daher elektronenmikroskopische Untersuchungen über den Attapulgit ausgeführt.

$$
\text { Vorbereitung der Proben }
$$

Als Rohstoff diente eine Fullererde der Fa. B e n s mann, Bremen, aus Florida mit der Bezeichnung XXF. Zur Prüfung der Einheitlichkeit wurde der Rohstoff in verschieden feine Fraktionen zerlegt. Die Gewinnung der Fraktionen verlief wie folgt:

Je $10 \mathrm{~g}$ Fullererde wurden in $1 l n / 10-\mathrm{Li}_{2} \mathrm{CO}_{3}$ dispergiert, mehrstündig geschüttelt, durch Zentrifugieren von überschüssigem Peptisationsmittel weitgehend befreit und mit destilliertem Wasser in Standzylindern aufgeschlämmt. Es wurden die Fraktionen $<1 \mu$, $<0,6 \mu,<0,3 \mu$ und $<0,1 \mu$ gewonnen.

Die Feinheiten dieser Fraktionen wurden aus Fallhöhe und Fallzeit, auf Grund der Stokesschen Formel für den Fall von kugelförmigen Körpern in Wasser, ermittelt.

Die Proben wurden aus folgenden Tiefen entnommen:

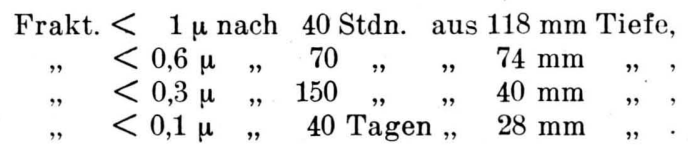

Da die Attapulgitteilchen sicher keine Kugeln sind, geben die Feinheitsangaben nicht absolute Werte, doch ist die Reihenfolge der Fraktionsbereiche als reell $\mathrm{zu}$ betrachten.

\section{Röntgenographische Untersuchung}

Zur Überprüfung der Homogenität der einzelnen Fraktionen wurden Proben der Suspensionen auf Membranfiltern abfiltriert und sowóhl im feuchten als auch im trockenen Zustand röntgenographisch untersucht. Es zeigte sich, daß sich das Röntgenbild bei allen Fraktionen von $<1 \mu$ bis $<0,1 \mu$ nicht änderte. Es ließen sich eindeutig nur die Interferenzen von Attapulgit erkennen. Die Verunreinigungen in der Roherde (Quarz, Kalkspat u.a.) waren also mit den Anteilen $>1 \mu$ abgetrennt worden. Die folgende Tabelle zeigt einen Vergleich der gemessenen Interferenzen der in dieser Arbeit untersuchten Fullererde mit den Werten von Brad ley, Lapparent und $\mathrm{K}$ e r r. Die Ubereinstimmung ist so befriedigend, daß das hier untersuchte Material zuverlässig als Attapulgit charakterisiert ist.

\section{Chemische Analyse}

Auch die Ubereinstimmung der analytischen Werte ist befriedigend. Ebenso die Übereinstimmung im Basenaustauschvermögen mit dem von Bradley ermittelten Wert (vergl. Tab. 2). 


\begin{tabular}{|c|c|c|c|c|c|}
\hline \multicolumn{2}{|c|}{$\begin{array}{l}\text { Fullererde XXF } \\
\qquad 1 \mu\end{array}$} & \multicolumn{2}{|c|}{$\begin{array}{c}\text { Bradley } \\
\text { Interferenzen der } \\
\text { hk0-Zone }\end{array}$} & \multirow{2}{*}{$\begin{array}{c}\text { Lap- } \\
\text { parent } \\
\text { d in } \AA\end{array}$} & \multirow{2}{*}{$\begin{array}{l}\mathrm{K} \text { e r r } \\
\mathrm{d} \text { in } \AA\end{array}$} \\
\hline Intensität & $\mathrm{d}$ in $\AA$ & $\mathrm{d}$ in $\AA$ & $\begin{array}{c}\text { Indi- } \\
\text { zierung }\end{array}$ & & \\
\hline s. st. & 10,5 & $10, \tilde{\mathfrak{n}}$ & 110 & 10,2 & - \\
\hline sch. & 6,42 & 6,44 & 200 & 6,4 & - \\
\hline sch. & 5,4 & 5,42 & $1: 30$ & 5,3 & - \\
\hline st. & 4,46 & 4.49 & 040 & 4,3 & 4,3 \\
\hline sch. & 4,14 & 4,18 & 310 & - & - \\
\hline s. sch. & 3,67 & 3,69 & 240 & - & - \\
\hline sch. & - & $3, \overline{0} 0$ & $33 \cup, 1$ วั0 & - & - \\
\hline s. sch. & 3,32 & - & - & - & - \\
\hline $\mathrm{m}$ & 3,22 & 3,23 & 400 & 3,25 & 3,25 \\
\hline s. sch. & $3,1) 3$ & 3,03 & $4 \cdot 20$ & - & - \\
\hline s. sch. & 2,77 & - & 350 & - & - \\
\hline st. & 2,56 & 2,61 & 440 & 2,55 & 2,55 \\
\hline sch. & - & 2,55 & 510 & - & - \\
\hline sch. & - & 2,38 & 530 & - & - \\
\hline sch. & 2,25 & - & 080 & - & - \\
\hline m. & 2,13 & 2,15 & 600 & 2,15 & 2,15 \\
\hline s. sch. & 1,82 & 1,82 & 390 & 1,8 & 1,8 \\
\hline sch. & 1,66 & 1.62 & 800 & 1,67 & 1,67 \\
\hline s. sch. & 1,54 & 1,56 & 680 & - & - \\
\hline $\mathrm{m}$ & 1,49 & 1,50 & 0120 & 1,49 & 1,49 \\
\hline s sch. & 1,36 & - & - & 1,37 & 1,37 \\
\hline s. sch. & 1,29 & - & - & 1,29 & 1,29 \\
\hline s. sch. & 1,245 & - & - & 1,25 & 1,25 \\
\hline
\end{tabular}

Tab 1. Interferenzen des Attapulgits.

s.st. = sehr stark, st. = stark, m. = mittel, sch. $=$ schwach, s.sch. $=$ sehr schwach

Elektronenoptische Untersuchung

Zur Untersuchung im Elektronenübermikroskop der Firma Sie mens \& Halske A.G. wurde jeweils ein Tropfen der feinen Suspension auf

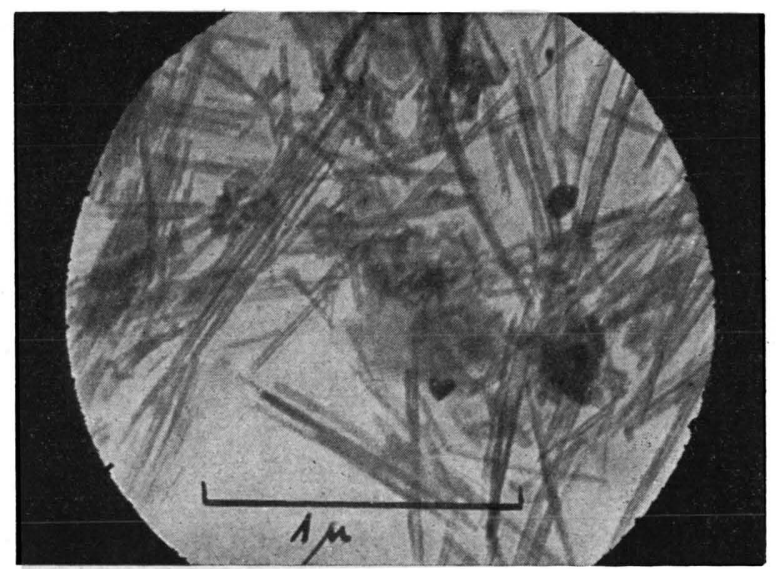

Abb. 1. Fullererde XXF. Fraktion $<1 \mu$. Vergr. $32500 \times$.

\begin{tabular}{|c|c|c|c|}
\hline \multicolumn{2}{|c|}{ Fullererde $\mathrm{XXF}^{*}$} & Lapparent & Bradley \\
\hline $\begin{array}{c}\text { Chemische } \\
\text { Zusammensetzg. }\end{array}$ & $\underset{\%}{<} \operatorname{lo}_{0} \mu$ & $\begin{array}{c}\text { Attapulgus } \\
\%\end{array}$ & $\begin{array}{c}\text { Attapulgus } \\
\%\end{array}$ \\
\hline $\mathrm{SiO}_{2} \ldots \ldots$ & 59,7 & 60,2 & 61,3 \\
\hline $\mathrm{TiO}_{2} \ldots \ldots$ & - & 0,8 & - \\
\hline $\mathrm{Al}_{2} \mathrm{O}_{3} \ldots \ldots$ & 10,1 & 9,83 & 11,4 \\
\hline $\mathrm{Fe}_{2} \mathrm{O}_{3} \ldots \ldots$ & 3,0 & 3,8 & 3,9 \\
\hline $\mathrm{FeO} \ldots \ldots$ & - & 0,3 & - \\
\hline $\mathrm{MnO} \ldots \ldots$ & - & 0,03 & - \\
\hline $\operatorname{MgO} \ldots \ldots$ & 9,4 & 10,1 & 11,7 \\
\hline $\mathrm{CaO} \ldots \ldots$ & 0,5 & 2,3 & - \\
\hline $\mathrm{Na}_{2} \mathrm{O} \ldots \ldots$ & 3,2 & 0,9 & - \\
\hline $\mathrm{K}_{2} \mathrm{O} \ldots \ldots$ & 0,7 & 0,8 & 0,5 \\
\hline $\mathrm{P}_{2} \mathrm{O}_{5} \ldots \ldots$ & - & 0,9 & - \\
\hline $\mathrm{H}_{2} \mathrm{O} \ldots \ldots$ & 12,6 & 10,2 & 10,8 \\
\hline Summe . . . . & 99,2 & 100,16 & 99,6 \\
\hline $\left.\begin{array}{l}\text { Basen- } \\
\text { austausch- } \\
\text { rermögen } \\
\text { mval/100 g }\end{array}\right\}$ & 21 & - & 21 \\
\hline Spezif. Gew. & 2,63 & - & - \\
\hline
\end{tabular}

* Die Werte wurden von Dr. E. M a e g d e f r a u im Chem. Inst. der Universität Rostock bestimmt.

Tab. 2. Analyse des Attapulgits bei $105^{\circ}$ getrocknet.

dem Zaponlackfilm der Objektblende eingedunstet und das Präparat bei einer Beschleunigungsspannung von $85 \mathrm{KV}$ belichtet und photographiert. Es zeigte sich, daß alle elektronenmikroskopischen Aufnahmen der verschiedenen Fraktionen nur feine Fasern erkennen ließen. Innerhalb des untersuchten Bereiches der Fraktionen von $<1 \mu$ bis $<0,1 \mu$ konnte kein deutlicher Unterschied in der

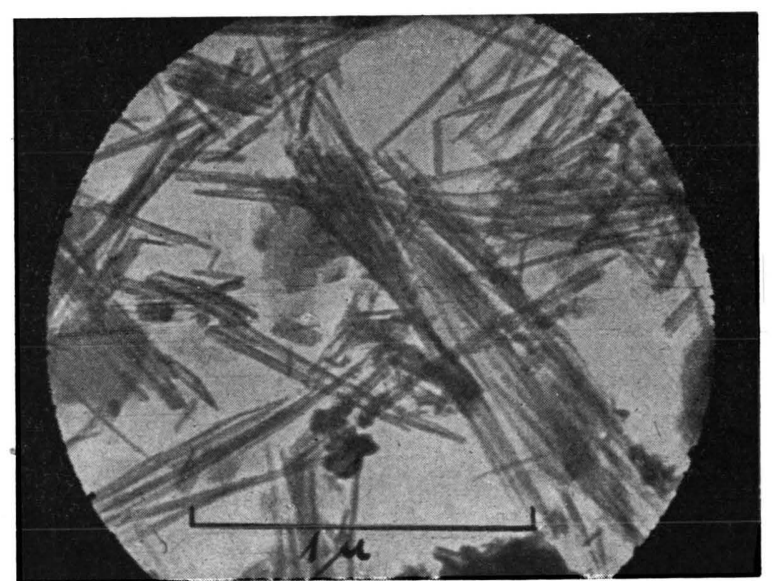

Abb. 2. Fullererde XXF. Fraktion $<0,1 \mu$. Vergr. $35000 \times$. 
Stärke oder Länge der Fasern erkannt werden.

Bei einer früher durchgeführten elektronenmikroskopischen Untersuchung von Tonen ${ }^{10}$ wurden bei einer rohen Fullererde neben den Fasern wolkige Gebilde beobachtet. Die feinen Fraktionen weisen diese Gebilde nicht auf. Es wird sich daher wohl um noch unreine Präparate gehandelt haben.

Die auf den Fasern erkennbaren Strukturlinien dürfen wahrscheinlich nicht mit deren Aufbau in Zusammenhang gebracht werden, sondern sind -wohl eher auf elektronenoptische Beugungseffekte zurückzuführen ${ }^{11}$. Die Fasern des Attapulgits zeigen im Elektronenübermikroskop Durchmesser von 250 bis $50 \AA$. Bei so geringer Dicke der Fasern ist ein Aufbau aus Kristallplättchen mit Schichtstruktur höchst unwahrscheinlich. Das Elektronenübermikroskop beweist also die Richtigkeit der Kristallstrukturbestimmung von Bradley, nach der die Kristalle des Attapulgits Faserstruktur besitzen.

10 J. Endell, Ber. dtsch. keram. Ges. 25, 113 [1944].
Bleichwirkung der Fullererde

Es liegt nahe, diese Faserstruktur mit der Bleichwirkung der amerikanischen Fullererde in Verbindung zu bringen. Um so mehr, als diese Fullererde im natürlichen Zustand bleichend wirkt, im Gegensatz zu den Montmorillonit enthaltenden Bleicherden, die ihre der Fullererde überlegene Bleichkraft erst nach Aufschließen mit Salzsäure erhalten, wobei die ursprüngliche Struktur angegriffen wird. Rechnet man mit einem mittleren Durchmesser der Fasern von $100 \AA$ und dem spezif. Gewicht von 2,63, so ergibt sich eine Oberfläche von $150 \mathrm{~m}^{2} / \mathrm{g}$. Dieser Wert kommt in die Nähe der Oberfläche einer Aktivkohle $\left(500 \mathrm{~m}^{2} / \mathrm{g}\right)$ ! Diese große freiliegende Oberfläche läßt die gute Bleichwirkung leicht verständlich erscheinen und gibt auch genügend Platz für das Basenaustauschvermögen von $21 \mathrm{mval} / 100 \mathrm{~g}$.

Für wertvolle Hinweise ist Verf. seinem verehrten Lehrer Hrn. Prof. Dr. Ulrich Hofman n zu Dank verpflichtet.

11 Vergl. E. Ruska, Kolloid-Z. 105, 43 [1943]; H. B o e r s c h, Kolloid-Z. 106, 169 [1944].

\title{
Über aktive $\gamma$-Aluminium-Oxyde*
}

\author{
Nach Versuchen von Walter Ste in e r \\ Von Robert Fricke
}

Aus dem Laboratorium für anorganische Chemie der Technischen Hochschule Stuttgart

(Z. Naturforschg. 1, 649 [1946]; eingegangen am 27. Februar 1946)

$\mathrm{N}$ achdem in einer früheren Arbeit ${ }^{1}$ bei in bestimmter Weise hergestellten $\gamma$-AluminiumOxyden neben schwammartiger Struktur ${ }^{2}$ und äußerst geringer Primärteilchengröß $\mathrm{e}^{3}$ auch z. Tl. recht erhebliche unregelmäßige Gitterstörungen gefunden worden waren, gelang es weiterhin, mit einer sehr vervollkommneten Methode der Lösungscalorimetrie $^{4}$ die Lösungswärmen dieser Präparate in Flußsäure auf $\pm 0,2 \%$ genau zu bestimmen.

Hierbei zeigte sich, daß zwischen einem bei $900^{\circ}$ und einem bei $500^{\circ}$ (jeweils 2 Stdn. Erhitzung) aus jungem Bayerit gewonnenen $\gamma$-Aluminium-Oxyd ein Unterschied in der Lösungs-

* 79. Mitteilung von R. Fri c ke u. Mitarbeitern über aktive feste Stoffe; 78. Mitt. im Druck bei der Z. anorg. allg. Chemie.

1 G. Weitbrecht u. R. Fricke, Z. anorg. allg. Chem. 253, 9 [1945].

2 Vergl. auch Th. Schoon u. E. Beger, Z. physik. Chem. A 189, 171 [1941]. wärme von rund 10 kcal pro Mol bestand, während die bei dazwischenliegenden Temperaturen hergestellten Präparate auch dazwischenliegende Lösungswärmen besaßen.

Die gefundene außerordentlich starke Erhöhung des Wärmeinhaltes der bei niedrigeren Temperaturen hergestellten Präparate läßt sich nicht durch die gefundenen Unterschiede in den mittleren Teilchengrößen (etwa zwischen 65 und $35 \AA$ bei den beiden Extrempräparaten) deuten. Się stehen aber in größenordnungsmäßiger Übereinstimmung mit den Wärmeinhalten, welche man aus den röntgenographisch quantitativ festgelegten unregelmäßigen Gitterstörungen ${ }^{\mathbf{1 , 5}}$ an Hand des Ganges der spezifischen Wärmen berechnen kann ${ }^{5}$.

3 s. a. R. Fricke, F. Niermann u. Ch. Feichtner, Ber. dtsch. chem. Ges. 70, 2318 [1937]

4 Erscheint demnächst in ds. Z.; Dissertat. W.St e i n e r, Stuttgart 1945.

5 R. Fricke u. E. Gw inner, Z. physik. Chem. A 183, 165 [1939]. 